\title{
Experimental evaluation and model of a nonlinear absorber for vibration attenuation
}

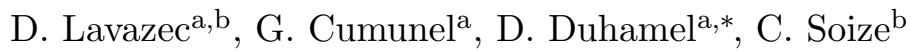 \\ ${ }^{a}$ Université Paris-Est, Laboratoire Navier, ENPC/IFSTTAR/CNRS \\ 6 et 8 Avenue Blaise Pascal, Cité Descartes, Champs-sur-Marne, 77455 \\ Marne-La-Vallée Cedex 2, France \\ ${ }^{b}$ Université Paris-Est, Laboratoire Modélisation et Simulation Multi Echelle, MSME \\ UMR 8208 CNRS \\ 5 bd Descartes, 77454 Marne-la-Vallée, France
}

\begin{abstract}
Because of their large wavelength, the noise and the vibrations at low frequencies cannot easily be reduced in the structures by using dissipative materials contrarily to the waves at middle and high frequencies. A possible technique for obtaining an attenuation is to randomly distribute absorbers in a matrix to attenuate acoustic waves and vibrations in such a low-frequency band. If linear absorbers are used, then it is difficult to obtain an attenuation on a broad low-frequency band with a reasonable number of absorbers. On the other hand, it is known that nonlinear absorbers allow the absorption bandwidth to be increased. However, such technique requires to properly design such a nonlinear absorber and to perform its experimental validation. The objective of the paper is to present the design of such an absorber with nonlinear geometrical effects and its experimental evaluation. It is experimentally proved that this nonlinearity type induces an attenuation on a broad frequency band around its first resonance. The novelty of this work is to present the design of an inclusion that has the potentiality to be reproduced at any scale, in particular for microstructured materials. For such an inclusion, we present its mechanical model for the simulation, its design, its manufacturing that is realized with a $3 \mathrm{D}$-printing system, the experiments, and the experimental identification of the model. The results show that a significant attenuation is effectively obtained over a broad low-frequency
\end{abstract}

\footnotetext{
${ }^{*}$ Corresponding author: D. Duhamel, denis.duhamel@enpc.fr

Email addresses: deborah.lavazec@univ-paris-est.fr (D. Lavazec), gwendal.cumunel@enpc.fr (G. Cumunel), denis.duhamel@enpc.fr (D. Duhamel ), christian.soize@univ-paris-est.fr (C. Soize)
} 
band, as intended.

Keywords: nonlinear absorption, vibrations control, noise control, homogenization, metamaterials

\section{Introduction}

At middle and high frequencies, the reduction of acoustic waves and vibration in structural elements can be obtained by using adapted poroelastic dissipative materials. However, for low frequencies, the wavelength are larger than the pores, and dissipative materials are less efficient. To get around this problem at low frequencies, oscillators-based absorbers have been designed in order to pump the vibratory energy. This type of technologies has extensively been studied in the litterature. Hereinafter, we present a non-exhaustive short bibliographical summary. Among the first papers devoted to the enery pumping by simple oscillators, the work by Frahm [1] in 1911 can be cited, in which the author proposes to add an auxiliary body to a structure to damp or avoid the vibration due to periodic impacts. The resonance vibrations of the main body are annulled by the secondary resonance vibrations of the smaller auxiliary body. In nearer years, tuned-mass dampers have been studied. The principle is to add an oscillator (generally a mass-spring-damper system) to a main structure that exibits an annoying resonance. The eigenfrequency of the damped oscillator is then adjusted for attenuating the resonance amplitude. With that, the peak is splited into two peaks of low amplitudes. A review made by Gutierrez Soto et al [2] presents a representative study on tuned-mass dampers. Metamaterials have also been used for the reduction of noise and vibration. A metamaterial is generally a composite for which these mechanical properties do not exist at the natural state. For instance, there are materials with both negative permittivity and negative permeability such as in [3]. In the framework of the absorption of vibration and noise, numerous papers have been published with metamaterials, see for instance $[4,5]$ for review papers. For instance metamaterials exhibiting a negative refracting index in the field of optics has been presented by [6] and equivalent negative mass in the field of acoustic material by $[7,8,9,10,11,12]$. Another works in the field of acoustic metamaterial can be found in [13] for acoustic cloaking, in the field of vibration attenuation with linear multiple discrete oscillators $[14,15,16]$ or multiple oscillators induced by continuous systems $[17,18,19,20,21]$ have presented metamaterials with linear behaviors. 
In 1952, Roberson [22] has presented the equations of a nonlinear dynamic vibration absorber and highlighted the fact that an auxiliary body with nonlinear dynamical behavior offers significant advantages over a linear absorber. Then, some works have been performed for analyzing vibration of nonlinear oscillators in a stochastic framework, such as in $[23,24,25,26]$ in which it is demonstrated the role plays by the nonlinearities in the transfer of the vibratory energy over a broad frequency band. Another works concerning the energy pumping by discrete nonlinear mechanical oscillators with linear damping without experimental results have been published such as $[27,28,29,30,31,32,33,34,35]$, and with nonlinear damping [36].

The work presented in this paper has been developed in the framework of the reduction of vibration and induced noise in structures at macro-scale for low frequencies for which the first structural modes are excited. The final objective of this work is to reduce vibrations and induced noise on a broad low-frequency band by using a microstructured material by inclusions that are randomly arranged in the material matrix. The novelty of this work is to present the design of an inclusion that has the potentiality to be reproduced at any scale, in particular for microstructured materials for which both the modelling approach and the parametric experiments performed for several structures manufactured with a 3D-printer, see [37] for a first example of this approach. The first step of this work is to design and to analyze the efficiency of an inclusion, which is made up of a cantilever beam with a mass at its end. This inclusion behaves as a nonlinear oscillator due to nonlinear geometrical effects, which is designed so that the energy pumping is effective on a broad frequency band around its resonance instead of a narrow frequency band as for an equivalent linear oscillator. The objectives of the paper are (1) to design the nonlinear oscillator in terms of form, dimension, and materials, in order that the vibratory energy is pumped on a broad frequency band around its first resonance, (2) to experimentally manufacture this system with a 3D-printing system, (3) to perform parametric experiments allowing to understand the bandwidth absorption versus the nonlinear amplitude levels, (4) to develop stochastic mechanical model to predict the responses, and (5) to perform the identification of the mechanical model with the experiments. The results that will be obtained will exhibit the physical attenuation over a broad low-frequency band, which is expected. Such a validated mechanical model is useful for designing a structural element including a random distribution of such a nonlinear oscillator. Compared to the communication [37], published in a French congress, this article develops the bibliography and the design of the inclusion. Above 
all more experimental results are presented in this article and are discussed in much more details. New and more detailed experimental results are the true novelty of this article which can be interesting for people working in this field.

In Section 2, the mechanical model of the inclusion and the related stochastic solver are introduced. In Section 3 the experimental design is performed in order to obtain an effective attenuation in the frequency band of analysis. The experimental measurements and the identification of the mechanical model are presented in Section 4. The last section is devoted to the conclusions on the results obtained and to the perspectives of the future work.

\section{Construction of a nonlinear model for representing the ab- sorber and stochastic solver}

\subsection{Model of the nonlinear oscillator}

As explained in Section 1, a nonlinear oscillator with one-DOF is proposed to model the nonlinear dynamical behavior of the inclusion. The oneDOF nonlinear model is composed of a mass-spring-damper system with a nonlinear spring and a nonlinear damper, subjected to an excitation of its base (see the scheme displayed in Figure 1). We introduce a parameterized family of one-DOF nonlinear oscillators, for which the proposed algebraic model is inspired/coherent with the linear viscoelasticity theory in finite displacements (nonlinear model) without memory [38, 39]. The motivation of this choice is the following: the nonlinear absorber that will be designed will not have material nonlinearities but only nonlinear geometrical effects and will have a viscoleastic type behavior related to the choice of the material. This is the reason why we have constructed a model coherent with the finite viscoelasticity theory.

In addition, we want that the stochastic response of the one-DOF nonlinear oscillator submitted to a centered stochastic excitation be a centered stochastic process. This constraint will be taken into account in the construction of the nonlinear damping force and of the nonlinear stiffness force deduced from the viscoelasticity theory in finite displacements. Let $X_{\text {imp }}^{\exp }(t)$ be the displacement imposed at the support in the absolute frame and let $X_{s}(t)$ be the relative displacement of the point mass with respect to the support. Let $\left\{\ddot{X}_{\text {imp }}^{\exp }(t), t \in \mathbb{R}\right\}$ be the acceleration imposed to the support, which is assumed to be a Gaussian stationary second-order centered stochastic process, defined on the probability space $(\Theta, \mathcal{T}, \mathcal{P})$, for which the power spectral 


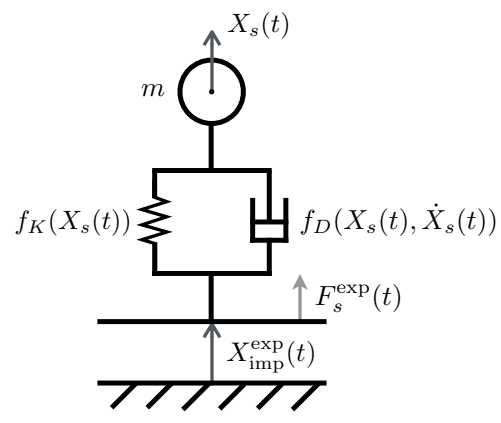

Figure 1: 1D simplified model.

density function is denoted by $S_{\ddot{X}_{\text {imp }}^{\exp }}(\omega)$. We are interested in finding the non-Gaussian stationary second-order stochastic process $\left\{X_{s}(t), t \in \mathbb{R}\right\}$ that satisfies, for all $t$ in $\mathbb{R}$, the following stochastic nonlinear equation

$$
m\left(\ddot{X}_{s}(t)+\ddot{X}_{\mathrm{imp}}^{\exp }(t)\right)+f_{D}\left(X_{s}(t), \dot{X}_{s}(t)\right)+f_{K}\left(X_{s}(t)\right)=0,
$$

which is rewritten as

$$
m \ddot{X}_{s}(t)+f_{D}\left(X_{s}(t), \dot{X}_{s}(t)\right)+f_{K}\left(X_{s}(t)\right)=F_{s}^{\exp }(t),
$$

in which the applied stochastic force $F_{s}^{\exp }(t)$ is written as

$$
F_{s}^{\exp }(t)=-m \ddot{X}_{\mathrm{imp}}^{\exp }(t),
$$

and where the nonlinear damping force $f_{D}$ and the nonlinear stiffness force $f_{K}$ are written as

$$
\begin{aligned}
f_{D}\left(X_{s}(t), \dot{X}_{s}(t)\right) & =\left(c_{1}+c_{2}\left|X_{s}(t)\right|\right) \dot{X}_{s}(t), \\
f_{K}\left(X_{s}(t)\right) & =k_{1} X_{s}(t)+k_{3}\left(X_{s}(t)\right)^{3} .
\end{aligned}
$$

The absolute displacement $X_{a}$ of the mass is such that

$$
X_{a}(t)=X_{s}(t)+X_{\mathrm{imp}}^{\mathrm{exp}}(t) .
$$

In these equations, $m$ is the participant mass of the inclusion, associated with the one-DOF oscillator, $c_{1}$ and $c_{2}$ are the two parameters describing the family of nonlinear damping force, and $k_{1}$ and $k_{3}$ are the two parameters related to the nonlinear stiffness force. It should be noted that the 
algebraic expression of the nonlinear terms that comes from the viscoelasticity theory without memory in finite displacements, should induce, for the nonlinear stiffness force, linear, quadratic, and cubic terms, corresponding to the nonlinear geometrical effect, and for the nonlinear damping force, a linear function of the velocity with a nonlinear coefficient of the displacement. Due to the constraint that we have introduced (centered response for a centered excitation), the quadratic term in the stiffness force must vanish and the nonlinear coefficient of the displacement in the damping force must be an even function of the displacement.

As we are interested in quantifying the vibratory energy absorbed by the oscillator, we introduced the mean input power $\Pi_{\text {in }}$ and the mean power dissipated $\Pi_{\text {diss }}$, which are such that

$$
\Pi_{\mathrm{in}}=E\left\{F_{s}^{\exp }(t) \dot{X}_{s}(t)\right\}, \quad \Pi_{\mathrm{diss}}=E\left\{f_{D}\left(X_{s}(t), \dot{X}_{s}(t)\right) \dot{X}_{s}(t)\right\}
$$

in which $E$ is the mathematical expectation. Since the stochastic processes $F_{s}^{\exp }$ and $\dot{X}_{s}$ are stationary, these two quantities are independent of time and can be rewritten

$$
\Pi_{\mathrm{in}}=\int_{\mathbb{R}} \pi_{\mathrm{in}}(\omega) d \omega, \quad \Pi_{\mathrm{diss}}=\int_{\mathbb{R}} \pi_{\mathrm{diss}}(\omega) d \omega,
$$

in which the density $\pi_{\text {in }}(\omega)$ and $\pi_{\text {diss }}(\omega)$ are such that

$$
\pi_{\mathrm{in}}(\omega)=S_{F_{s}^{\exp } \dot{X}_{s}}(\omega) \quad, \quad \pi_{\mathrm{diss}}(\omega)=S_{f_{D} \dot{X}_{s}}(\omega) .
$$

In Eq. (7), $S_{F_{s}^{\text {exp }} \dot{X}_{s}}$ is the cross-spectral density function of the stationary stochastic processes $\left\{F_{s}^{\exp }(t), t \in \mathbb{R}\right\}$ and $\left\{\dot{X}_{s}(t), t \in \mathbb{R}\right\}$, and $S_{f_{D} \dot{X}_{s}}(\omega)$ is the cross-spectral density function of the stationary stochastic processes $\left\{f_{D}\left(X_{s}(t), \dot{X}_{s}(t)\right), t \in \mathbb{R}\right\}$ and $\left\{\dot{X}_{s}(t), t \in \mathbb{R}\right\}$. The frequency-dependent energy pumping is therefore characterized by $\pi_{\text {diss }}(\omega)$, which is equal to $\pi_{\text {in }}(\omega)$ due to the power balance equation of the stochastic system. In order to qualify the efficiency of the energy pumping as a function of the intensity of the nonlinearity that will be characterized by the value of the dimensionless response amplitude defined in Section 4.2, we introduce the normalized quantity,

$$
\pi_{\mathrm{in}, \mathrm{norm}}(\omega)=\frac{\pi_{\mathrm{in}}(\omega)}{S_{F_{s}^{\exp }(\omega)}}
$$

In order to experimentally identify the vector-valued parameter of the model, denoted by $\mathbf{w}=\left(c_{1}, c_{2}, k_{1}, k_{3}\right)$, which belongs to an admissible set $\mathcal{C}_{\text {ad }}$, we 
introduce the frequency dependent function $\operatorname{FRF}(\omega ; \mathbf{w})^{2}$, defined on $B_{o}$ by

$$
\operatorname{FRF}(\omega ; \mathbf{w})^{2}=\frac{\mid S_{\left.\dot{X}_{s} F_{s}^{\exp }(\omega)\right|^{2}}}{\mid S_{\left.F_{s}^{\exp }(\omega)\right|^{2}}}=\left|\pi_{\text {in,norm }}(\omega)\right|^{2},
$$

in which the w-dependence in the right-hand side of Eq. (9) has been removed.

It should be noted that if $f_{D}(x, \dot{x})=c_{1} \dot{x}$ and $f_{K}(x)=k_{1} x$, the model corresponds to the one of a linear oscillator and $\mathrm{FRF}^{2}$ represents the square of the modulus of the frequency response function of the associated linear filter for which $F_{s}^{\exp }$ is the input and $\dot{X}_{s}$ is the output.

\subsection{Stochastic solver and signal processing}

Stochastic solver. For constructing the stationary stochastic solution of the nonlinear differential equation defined by Eq. (1), the Monte Carlo method (see [40]) is used. Let $\left\{F_{s}^{\exp }\left(t ; \theta_{\ell}\right), t \in \mathbb{R}\right\}$ be a realization of the stochastic process $F_{s}^{\text {exp }}$ for $\theta_{\ell}$ in $\Theta$. We consider $L$ independent realizations $\theta_{\ell}=1, \ldots, L$ in $\Theta$ constructed with the generator described in a next paragraph of this section. For each realization $\theta_{\ell}$, we would have then to solve the deterministic nonlinear differential equation associated with stochastic Eq. (1): calculate $X\left(t ; \theta_{\ell}\right)$ for all $t$ in $\mathbb{R}$ such that

$$
m \ddot{X}\left(t ; \theta_{\ell}\right)+f_{D}\left(X\left(t ; \theta_{\ell}\right), \dot{X}\left(t ; \theta_{\ell}\right)\right)+f_{K}\left(X\left(t ; \theta_{\ell}\right)\right)=F_{s}^{\exp }\left(t ; \theta_{\ell}\right) .
$$

This deterministic Eq. (10) cannot numerically be solved because $t$ belongs to an infinite interval. Consequently, this problem is replaced by the following approximate problem with initial conditions over a finite interval of length $T$ : calculate $X\left(t ; \theta_{\ell}\right)$ for $t$ belonging to the finite interval $\left.] 0, T\right]$, solution of Eq. (10) with the initial conditions at time $t=0$,

$$
X\left(0, \theta_{\ell}\right)=0 \quad, \quad \dot{X}\left(0, \theta_{\ell}\right)=0 .
$$

With such initial conditions, the transient part of the response would be 0 for a linear second-order dynamical system and it is assumed to be 0 or negligeable for the family of the nonlinear second-order dynamical systems that are considered (we have effectively checked the validity of these hypotheses for the numerical application presented in the paper). The part of the trajectory on $[0, T]$ corresponds to the stationary response, $X_{s}\left(t ; \theta_{\ell}\right) \simeq X\left(t ; \theta_{\ell}\right)$ for $t$ in $[0, T]$. Time $T$ that is related to the frequency resolution is defined in a next paragraph. The deterministic problem defined by Eq. (10) for $t$ in $] 0, T]$ with the initial conditions defined by Eq. (11) for $t=0$ will 
be solved by using the Störmer-Verlet scheme presented in a next paragraph.

Time and frequency sampling. For solving the nonlinear deterministic equation with initial conditions associated with a given realization $\theta_{\ell}$, we need to generate realizations of the Gaussian stationary stochastic process $F_{s}^{\exp }$ on the time interval $[0, T]$. Consequently, the time and the frequency samplings are constructed with respect to this time interval. The signal processing requires a time sampling with a constant time step $\Delta_{t}$ that is performed using the Shannon theorem for the stationary stochastic processes [41]. The sampling frequency is $f_{e}=2 f_{\max }$ where $f_{\max }$ is the maximum frequency that exists in the frequency band of analysis and the time step is $\Delta_{t}=1 / f_{e}$. The corresponding time sampling is $t_{\alpha}=\alpha \Delta_{t}$ with $\alpha=0,1, \ldots, N-1$ in which the integer $N$ is chosen in order that the time duration $T=8 \mathrm{~s}$, with $T=N \Delta_{t}$ yielding $N=16,384$. The frequency resolution $\Delta_{f}=1 / T=$ $0.125 \mathrm{~Hz}$. The corresponding sampling points in the frequency domain are $f_{\beta}=-f_{\max }+(\beta+1 / 2) \Delta_{f}$ for $\beta=0,1, \ldots, N-1$.

Generation of independent realizations of Gaussian stationary stochastic process $F_{s}^{\text {exp }}$. The usual second-order spectral representation of the stationary stochastic processes is used $[42,43]$. The power spectral density function

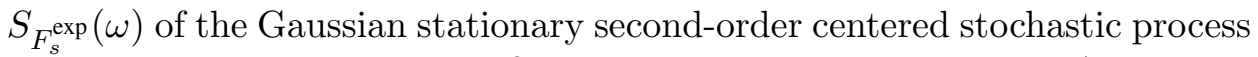
$F_{s}^{\text {exp }}$ is such that $S_{F_{s}^{\exp }}(\omega)=m^{2} S_{\ddot{X}_{\text {imp }}^{\exp }}(\omega)$, in which $S_{\ddot{X}_{\operatorname{imp}}^{\exp }}(\omega)=\omega^{4} S_{X_{\text {imp }}^{\exp }}(\omega)$. The autocorrelation function $\tau \mapsto R_{\ddot{X}_{\text {imp }}^{\exp }}(\tau)$ of stationary stochastic process $\ddot{X}_{\text {imp }}^{\exp }$ is such that $R_{\ddot{X}_{\text {imp }}^{\exp }}(\tau)=E\left\{\ddot{X}_{\text {imp }}^{\exp }(t+\tau) \ddot{X}_{\text {imp }}^{\exp }(t)\right\}$ and $R_{\ddot{X}_{\text {imp }}^{\exp }}(\tau)=$

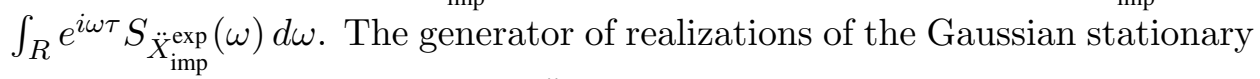
second-order stochastic process $\ddot{X}_{\text {imp }}^{\text {exp }}$ is based on the usual spectral representation (see $[44,45])$. Let $\psi_{0}, \ldots, \psi_{N-1}$ be $N$ mutually independent uniform random variables on $[0,1]$ and let $\phi_{0}, \ldots, \phi_{N-1}$ be $N$ mutually independent uniform random variables on $[0,2 \pi]$, which are independent of $\psi_{0}, \ldots, \psi_{N-1}$. The spectral representation used is written, for all $t$, as

$$
\ddot{X}_{\text {imp }}^{\exp }\left(t ; \theta_{\ell}\right) \simeq \sqrt{2 \Delta_{\omega}} \operatorname{Re}\left\{\sum_{\beta=0}^{N-1} \sqrt{S_{\ddot{X}_{\text {imp }}^{\exp }\left(\omega_{\beta}\right)}} Z_{\beta} e^{-i \omega_{\beta} t} e^{-i \phi_{\beta}}\right\},
$$

with $\Delta_{\omega}=2 \pi \Delta_{f}$, where $Z_{\beta}=\sqrt{-\log \left(\psi_{\beta}\right)}$, where $\omega_{\beta}=2 \pi f_{\beta}$, and where Re denote the real part of complex numbers. From Eq. (12), it can be 
deduced that, for all $t$, the realization $\ddot{X}_{\mathrm{imp}}^{\exp }\left(t ; \theta_{\ell}\right)$ is written as

$$
\ddot{X}_{\text {imp }}^{\exp }\left(t ; \theta_{\ell}\right) \simeq \sqrt{2 \Delta_{\omega}} \operatorname{Re}\left\{\sum_{\beta=0}^{N-1} g_{\beta, \ell} e^{-i \omega_{\beta} t}\right\},
$$

in which $g_{\beta, \ell}=\sqrt{S_{\ddot{X}_{\text {imp }}^{\exp }\left(\omega_{\beta}\right)}} Z_{\beta}\left(\theta_{\ell}\right) e^{-i \phi_{\beta}\left(\theta_{\ell}\right)}$. In practice, for each $\ell$, Eq. (13) is computed for $t=t_{\alpha}$ with $\alpha=0, \ldots, N-1$. Consequently, the numerical calculation is done by using the FFT $\left\{\widehat{g}_{0, \ell}, \ldots, \widehat{g}_{N-1, \ell}\right\}$ of $\left\{g_{0, \ell}, \ldots, g_{N-1, \ell}\right\}$, which is such that $\widehat{g}_{\alpha, \ell}=\sum_{\beta=0}^{N-1} g_{\beta, \ell} \exp \{-2 i \pi \alpha \beta / N\}$ and which yields, for $\alpha=0,1, \ldots, N-1$,

$$
\ddot{X}_{\text {imp }}^{\exp }\left(t_{\alpha} ; \theta_{\ell}\right)=\sqrt{2 \Delta_{\omega}} \operatorname{Re}\left\{\exp \left\{-i \pi \alpha\left(\frac{1-N}{N}\right)\right\} \widehat{g}_{\alpha, \ell}\right\} .
$$

Störmer-Verlet integration scheme. The Störmer-Verlet integration scheme is well suited for the resolution of dynamical Hamiltonian systems [46, 47] and stays very efficient for dissipative Hamiltonian systems as proposed in [48]. Such a scheme preserves the mechanical energy during the numerical integration. Equation (10) with initial conditions defined by Eq. (11) is then written as the following first order differential equation, for $t$ in $] 0, T]$,

$$
\left\{\begin{array}{l}
\dot{X}\left(t ; \theta_{\ell}\right)=\frac{1}{m} Y\left(t ; \theta_{\ell}\right) \\
\dot{Y}\left(t ; \theta_{\ell}\right)=-f_{D}\left(X\left(t ; \theta_{\ell}\right), \frac{Y\left(t ; \theta_{\ell}\right)}{m}\right)-f_{K}\left(X\left(t ; \theta_{\ell}\right)\right)+F_{s}^{\exp }\left(t ; \theta_{\ell}\right) .
\end{array}\right.
$$

We use the notations $x_{\ell}^{\alpha}=X\left(t_{\alpha} ; \theta_{\ell}\right)$ and $y_{\ell}^{\alpha}=Y\left(t_{\alpha} ; \theta_{\ell}\right)$. The Störmer-Verlet integration scheme for Eq. (15) is then written, for $\alpha=0,1, \ldots, N-1$, as

$$
\left\{\begin{aligned}
x_{\ell}^{\alpha+1 / 2} & =x_{\ell}^{\alpha}+\frac{\Delta_{t}}{2 m} y_{\ell}^{\alpha} \\
y_{\ell}^{\alpha+1}= & y_{\ell}^{\alpha}+\Delta_{t}\left[-\frac{1}{2}\left(f_{D}\left(x_{\ell}^{\alpha+1 / 2}, y_{\ell}^{\alpha} / m\right)+f_{D}\left(x_{\ell}^{\alpha+1 / 2}, y_{\ell}^{\alpha+1} / m\right)\right)\right. \\
& \left.-f_{K}\left(x_{\ell}^{\alpha+1 / 2}\right)+F_{s}^{\exp }\left(t_{\alpha+1} ; \theta_{\ell}\right)\right] \\
x_{\ell}^{\alpha+1}= & x_{\ell}^{\alpha+1 / 2}+\frac{\Delta_{t}}{2 m} y_{\ell}^{\alpha+1}
\end{aligned}\right.
$$

in which $F_{s}^{\exp }\left(t_{\alpha+1} ; \theta_{\ell}\right)=-m \ddot{X}_{\text {imp }}^{\exp }\left(t_{\alpha+1} ; \theta_{\ell}\right)$ and where $x_{\ell}^{0}=y_{\ell}^{0}=0$. 
Signal processing. For estimating, the power spectral density functions and the cross-spectral density functions defined in Eqs. (7) and (9), the periodogram method [41] is used for which the rectangular time-window on ] $0, T]$ is used.

\section{Experimental design}

In Section 2, we have presented the nonlinear dynamical model of the inclusion and the related stochastic solver. In this section, we present the design of an inclusion whose response can be simulated with this nonlinear dynamical model.

Defining the constraints for the design of the inclusion and its consequences on the design. The following constraints have been taken into account for defining the design:

- The dimension of the inclusion has been setted at about two centimeters in order to be representative of the scheduled technology for which such inclusions would be incorporate in a composite material such as a concrete panel.

- The inclusion must have the capability to exhibit a strong nonlinear geometrical effect with reasonable finite displacements to remain in the bulk whose geometry has previously been defined.

- The inclusion must exhibit a relatively small damping in order to optimize the transmitted mechanical power to the nonlinear oscillator in order to optimize the absorption.

The first system that we have worked was made up of a point mass centered in a spherical steel shell with an internal elastomer layer around the mass. The main advantage of this system was to have an isotropic absorption property no matter the direction of excitation. The elastomer had a hyperelastic behavior and after experimental tests, we have rejected this system because of the important damping due to the elastomer. After that, we studied a system inspired by the nonlinear energy sinks (NES) composed of a point mass linked to two prestressed traction springs clamped at their ends. In this configuration, the springs had effectively a nonlinear behavior when the point mass is excited in the transverse direction. However, large displacements are needed for obtaining a significant geometrical nonlinear effect what was not possible. Then, we focused on two systems composed 
of a point mass attached to a beam. For the first one, the beam was biclamped and the point mass was located at its center. The second one was a cantilever beam with a point mass located at its free end. In these two cases, the excitation is transverse to the beam and the nonlinear behavior is obtained by the geometric nonlinearities of the beam submitted to finite displacements.

For limiting the developments in this paper, only the inclusion that has been designed with a cantilever beam and its point mass at its end is presented. The point mass $m$ of the inclusion is equal to the sum of the mass of the cube, with the mass of the accelerometer attached to it for the measurements and with the mass of the accelerometer cable.

Preliminary experiments have been performed with a monopoint velocimeter Polytech PDV-100 without accelerometer for testing possible damping and stiffness effects of the accelerometer cable. No significant effect has been found with respect to the material damping and structural stiffness by comparison with the same results obtained with the accelerometer.

Design of the inclusion. To determine the length $\ell$ of the beam, its bending stiffness $E I$, and the point mass $m$ of the inclusion, we have used the equation of the resonance frequency of a cantilever beam with a point mass at its end:

$$
f_{p}=\frac{1}{2 \pi} \sqrt{\frac{3 E I}{m \ell^{3}}},
$$

for which it is assumed that the mass of the beam is negligeable with respect of the point mass. The magnitudes of these parameters are shown in Table 1 , in which $f_{p}, \ell, E, d_{\max }$ have been defined taking into account the experimental constraints for the manufacturing of the inclusion and where $I$ has arbitrarily be fixed for limiting the number of design parameters.

\begin{tabular}{|c|c|}
\hline Parameters & Magnitudes \\
\hline \hline$f_{p}$ & $\leq 300 \mathrm{~Hz}$ \\
$\ell$ & $\leq 0.03 \mathrm{~m}$ \\
$I$ & $8.3 \times 10^{-14} \mathrm{~m}^{4}$ \\
$E$ & $\left\{10^{9}, 250 \times 10^{9}\right\} \mathrm{Pa}$ \\
$m$ & $\geq 4.4033 \times 10^{-6} \mathrm{~kg}$ \\
\hline
\end{tabular}

Table 1: Magnitudes of parameters for the design of the inclusion.

Material of the inclusion. The first idea for the material of the inclusion was 
to use metallic parts (steel, aluminum) for the beam and the point mass. However, it was difficult to correctly embed the point mass to the beam and the beam to its support. That is why we have chosen to use a 3D-printing system and to print the beam, its support, and the point mass in a single piece, using a polymer material.

Final design of the inclusion with its support. The inclusion is attached to a rigid frame for which the $\mathrm{CAO}$ of the final design is shown in Figure 8(a). The point mass of the inclusion is constituted of a cube, embedded at the free end of the beam. The other end of the beam is continuously connected to the frame. The beam length is $0.026 \mathrm{~m}$ and the width of its square section is around $0.001 \mathrm{~m}$. The width of the cube is about $0.008 \mathrm{~m}$. The point mass $m$ of the inclusion is equal to the sum of the mass of the accelerometer attached to it for the measurements $(0.4 \mathrm{~g})$, with the mass of the accelerometer cable $(0.2 \mathrm{~g})$, and with the mass of the cube $(0.55 \mathrm{~g})$, that is to say a total point mass of $1.2 \mathrm{~g}$ (the mass of the beam is neglected as we have assumed). The mass density of ABS is $1,780 \mathrm{~kg} / \mathrm{m}^{3}$. Some experimental traction tests have been carried out to identify the mechanical properties of ABS. The experiments give $2.2 \times 10^{9} \mathrm{~Pa}$ for the Young modulus and 0.35 for the Poisson coefficient. This inclusion has been designed so that the first eigenfrequency of the frame is $1,200 \mathrm{~Hz}$ and the first eigenfrequency of the inclusion around $24 \mathrm{~Hz}$, which shows that the frame can effectively be considered as rigid for analyzing the dynamical response of the inclusion in the observed frequency band $B_{o}$ that is centered around the first eigenfrequency.

\section{Experimental measurements and identification of the model}

\subsection{Manufacturing of the test inclusion}

The inclusion is manufactured using a 3D-printing system. The inclusion and the rigid frame on which the inclusion is attached, are printed in one piece in ABS (Acrylonitrile Butadiene Styrend) that is a material commonly used for 3D printing with the fused deposition modeling technique (see Figure 2(b)).

\subsection{Description of the experimental procedure}

We are interested in analyzing the stationary random response of the inclusion in the frequency band of analysis $B_{a}=\left[0, f_{\max }\right]$ with $f_{\max }=1,024 \mathrm{~Hz}$, induced by the stationary random excitation generated by an imposed acceleration of the embedded end of the beam via the rigid frame (see Figure 3). This acceleration is equal to the acceleration that is imposed to the frame 


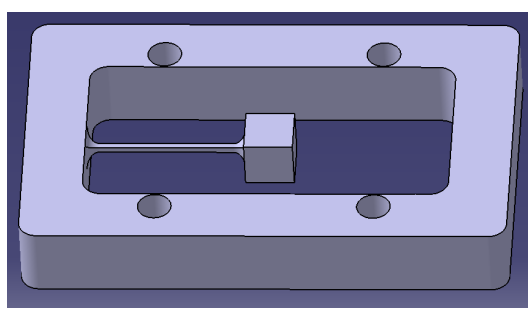

(a) $\mathrm{CAO}$

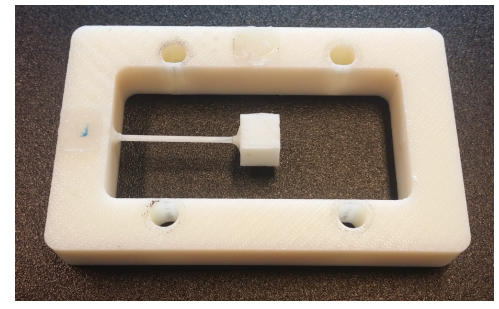

(b) 3D printing

Figure 2: $\mathrm{CAO}$ and 3D printing of the test structure.

(that can be considered as rigid in the observed frequency band), on which a stationary random external force is applied. The observed frequency band is $B_{o}=[21,26] H z \subset B_{a}$, which contains the resonance frequency for the all amplitudes that are considered for the excitation. A Gaussian stationary random excitation in the frequency bandwidth $[5-800] \mathrm{Hz}$ is applied, which is delivered by a shaker (Brüel \& Kjaër 4808) with a power amplifier (Brüel $\&$ Kjaër 2719). The excitation signal, $\ddot{X}_{\text {imp }}^{\text {exp }}$, is measured by an accelerometer (Brüel \& Kjaër 4507-B004) attached to the frame and the response $\ddot{X}^{\exp }$ of the inclusion point mass by an accelerometer Endevco 2250A-10 attached to the point mass (see Figure 3 ). We have chosen to attach an accelerometer to the point mass for measuring its response, in order to avoid some problems that would be induced by the use of different instrumentations adapted to different excitation levels. The sensors data are acquired with National Instrument Hardware. A CompactDAQ module 9234 is used for the measurements of the two IEPE accelerometers and a module 9263 is used for the signal generation of the excitation. The modules are used with a 4-slot USB chassis 9174 . The software used to process the measurements is SO Analyzer from $\mathrm{m}+\mathrm{p}$ international. The useful bandwidth is set to $1,024 \mathrm{~Hz}$ that leads us to a time sample rate of $2,048 \mathrm{~Hz}$. The block size is 16,384 points, which corresponds to an acquisition time of $8 s$ for a measurement block and so the frequency step is equal to $0.125 \mathrm{~Hz}$. The overlap factor is chosen equal to $66 \%$. The number of blocks that are processed is 101, which corresponds to a total acquisition time of $280 \mathrm{~s}$ for each excitation level. The $H_{1}$ estimator with Hanning window is used to estimate the FRF functions. The experimental configuration can be viewed in Figure 3. Experimental responses have been measured for seven excitation levels. These cases are identified by the dimensionless response amplitude $d / h$ in which $h$ is the thickness of the beam $(h=0.001 \mathrm{~m})$ and $d$ is defined as the statistical mean of the " peak-to-peak" deflection amplitudes relative to the frame obtained 
for each measurement block, such that, using Eq. (5),

$$
d=\frac{1}{2} E\left\{\max _{t}\left(X_{s}^{\exp }(t)\right)-\min _{t}\left(X_{s}^{\exp }(t)\right)\right\}
$$

where $X_{s}^{\exp }(t)=X_{a}^{\exp }(t)-X_{\text {imp }}^{\exp }(t)$. For instance, if $d / h=1$, the displacement of the inclusion is of the order of magnitude of the thickness of the beam. Seven values of $d / h$ are considered for the experiments and are, in percent, $1.9,11,52.3,82.4,96.7,131$, and 134 .

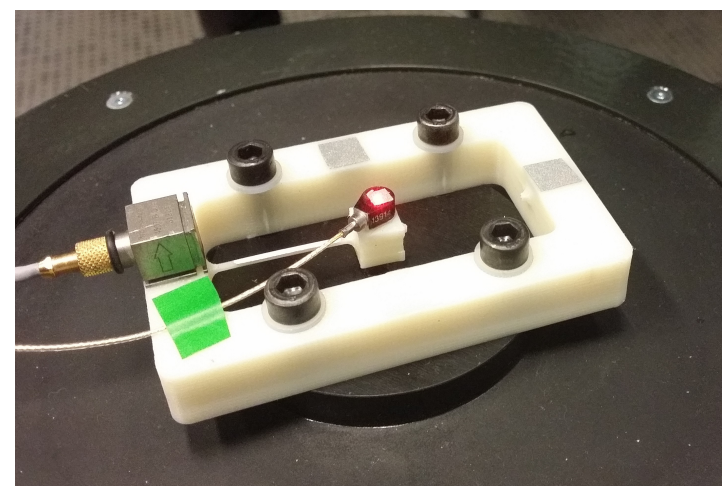

Figure 3: The experimental configuration.

\subsection{Experimental results}

The experimental power spectral density (PSD) functions $S_{F_{x}}^{\text {exp }}$ of the excitation are displayed in Figure 4 for the seven considered values of $d / h$. Figure 5 depicts the measured transmissibility functions obtained from the post-processing of the time data of the two accelerometers for each values of $d / h$. For each block, the displacement signals are obtained by numerical double integration of the acceleration signals. A high-pass filter is applied before each numerical integration. The square of the modulus $\operatorname{FRF}^{\exp }(2 \pi f)^{2}$ of the experimental frequency response function is displayed in Figure 6 and the experimental normalized input power density $\pi_{\mathrm{in}, \mathrm{erm}}^{\exp }(2 \pi f)$ is displayed in Figure 7 (which is the square root of $\operatorname{FRF}^{\exp }(2 \pi f)^{2}$ as noted in the Eq. (9)).

\subsection{Experimental identification of the model}

As explained in Section 2.1, for all the considered values of $d / h$, the experimental identification of the damping and stiffness parameters is performed by minimizing over the observed frequency band $B_{o}$, the distance 


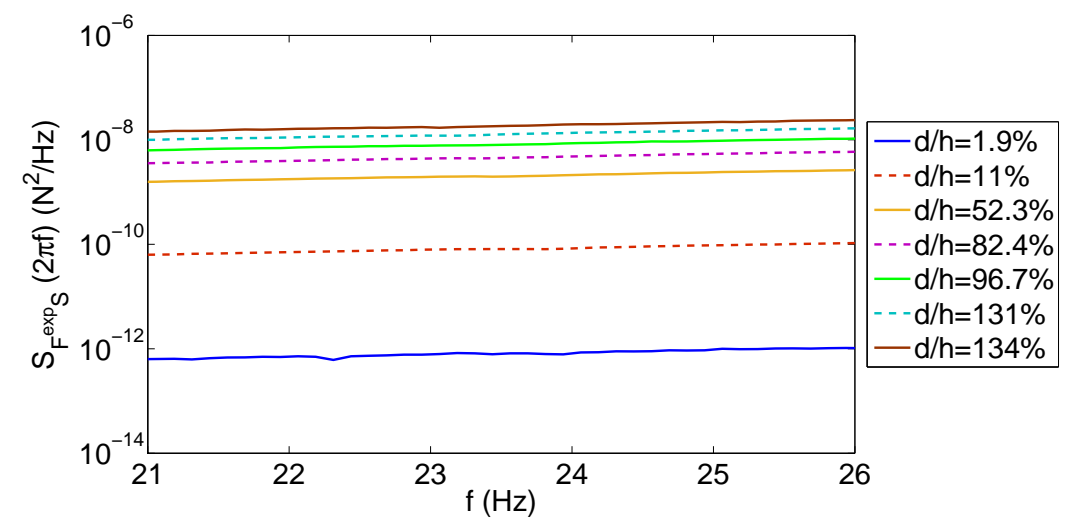

Figure 4: Experimental PSD functions $S_{F_{x}}^{\exp }$ of the excitation for the seven considered values of $d / h$.

between the function $\operatorname{FRF}(\omega, \mathbf{w})^{2}$ (defined by Eq. (9)) computed with the model and its experimental counterpart $\operatorname{FRF}^{\exp }(\omega)^{2}$ that is to say, by solving the following optimization problem,

$$
\mathbf{w}^{\text {opt }}=\arg \min _{\mathbf{w} \in \mathcal{C}_{\mathrm{ad}}} J(\mathbf{w})
$$

in which the objective function $J(\mathbf{w})$ is written as

$$
J(\mathbf{w})=\int_{B_{o}}\left|\operatorname{FRF}(\omega, \mathbf{w})^{2}-\operatorname{FRF}^{\exp }(\omega)^{2}\right| d \omega
$$

Note that the right-hand side member of the above equation is the $L^{1}$ norm of the function $\omega \mapsto \operatorname{FRF}(\omega, \mathbf{w})^{2}-\operatorname{FRF}^{\exp }(\omega)^{2}$. The optimal value $\mathbf{w}^{\mathrm{opt}}=\left(c_{1}^{\mathrm{opt}}, c_{2}^{\mathrm{opt}}, k_{1}^{\mathrm{opt}}, k_{3}^{\mathrm{opt}}\right)$ obtained is

$$
\left\{\begin{array}{l}
c_{1}^{\mathrm{opt}}=0.0038 \mathrm{Ns} / \mathrm{m} \\
c_{2}^{\mathrm{opt}}=10 \mathrm{Ns} / \mathrm{m}^{2} \\
k_{1}^{\mathrm{opt}}=26.8 \mathrm{~N} / \mathrm{m} \\
k_{3}^{\mathrm{opt}}=-4 \times 10^{6} \mathrm{~N} / \mathrm{m}^{3}
\end{array}\right.
$$

During the identification process, we have noted that the damping effect is effectively nonlinear, that is coherent with the nonlinear dynamical model introduced in Section 2. This damping nonlinearity can also be explained by the fact that the beam of the 3D-printing inclusion exhibits a stratified 


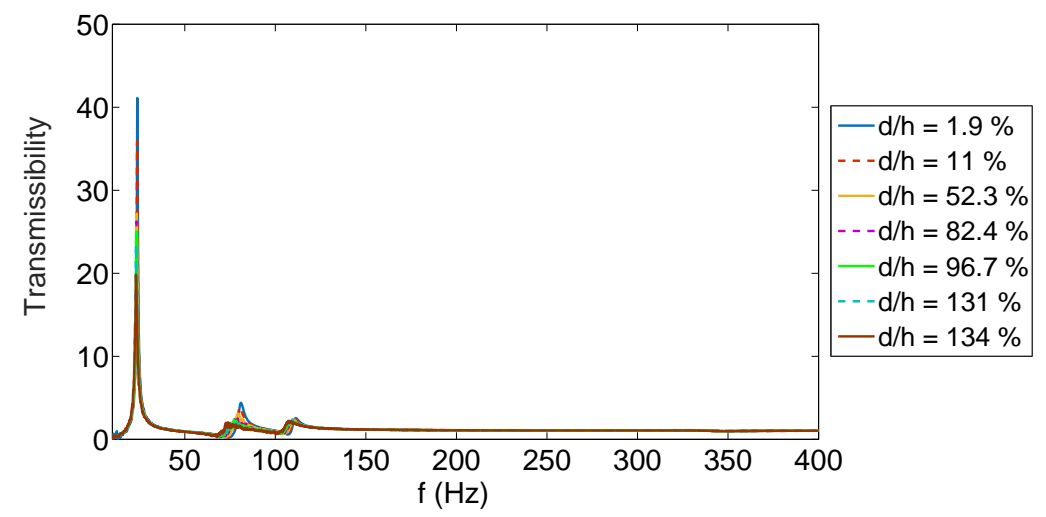

(a) Measured transmissibility for the considered values of $d / h$.

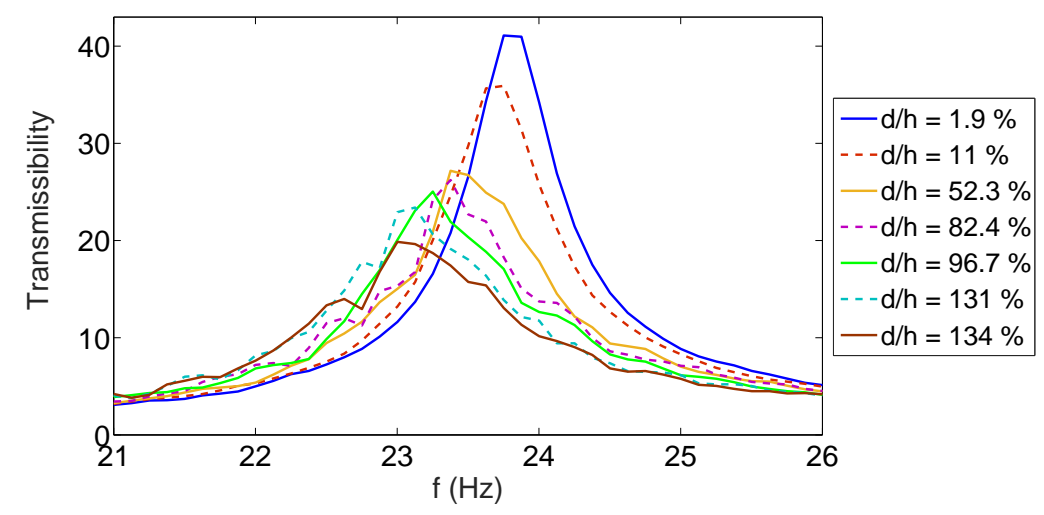

(b) Zoom around the first eigenfrequency of the measured transmissibility for the considered values of $d / h$.

Figure 5: Transmissibility functions for the different excitation levels of the inclusion base (the frame).

structure of layers with pores (miss of matter) generated by the 3D-printing process (Figure 8). For high response amplitudes, the shear deformation could induce small slides at the interface of the layers.

For each one of the seven values of $d / h$, Figure 9 displays the experimental function $\omega \mapsto \operatorname{FRF}^{\exp }(\omega)^{2}$ (upper figure) and the optimized function $\omega \mapsto \operatorname{FRF}\left(\omega, \mathbf{w}^{\text {opt }}\right)^{2}$ (lower figure) corresponding to the experimental identification of the model. The comparison of these two figures shows a reasonable 


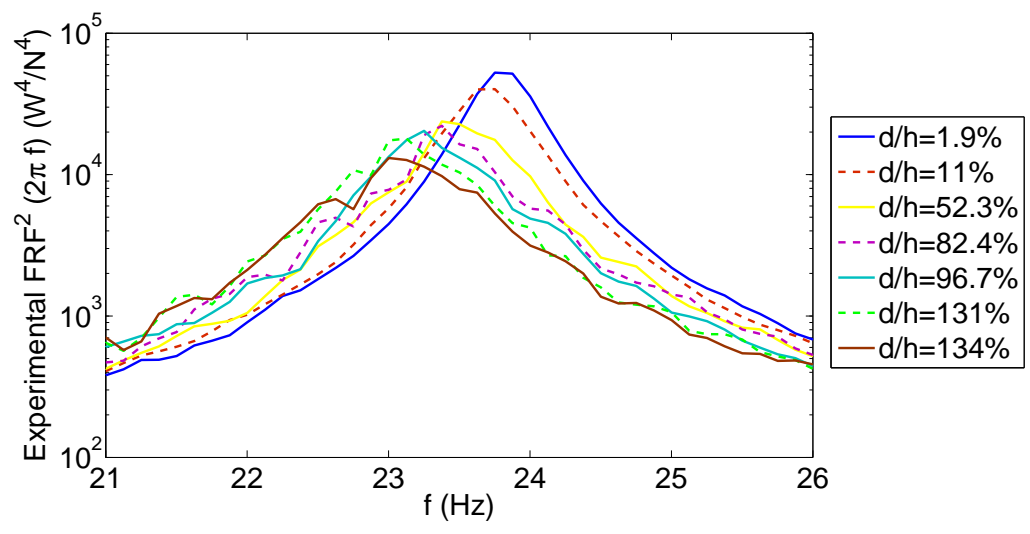

Figure 6: Square of the modulus $\operatorname{FRF}^{\exp }(2 \pi f)^{2}$ of the experimental frequency response function for the seven values of $d / h$.

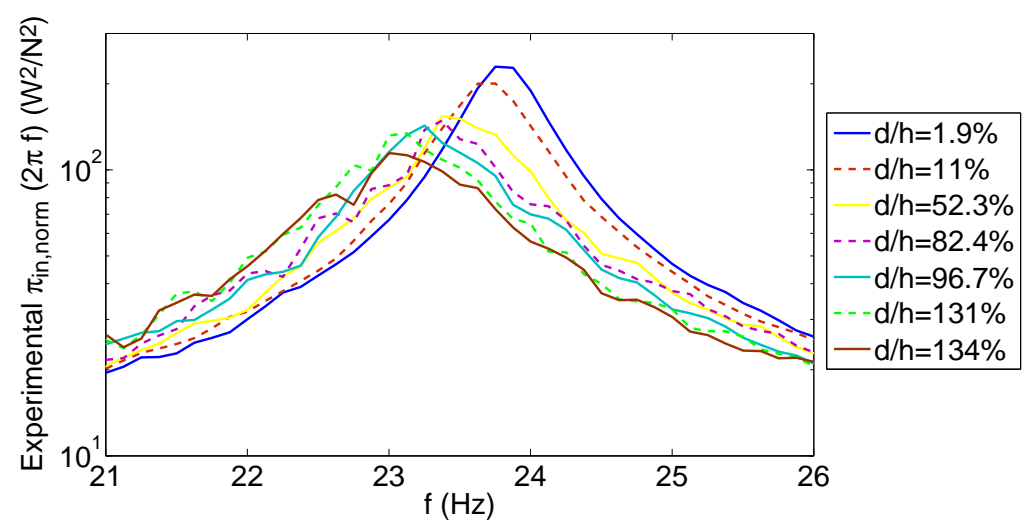

Figure 7: Experimental normalized input power density $\pi_{\text {in,norm }}^{\text {exp }}(2 \pi f)$ for the seven values of $d / h$.

agreement between the experiments and the identified model. The prevision could certainly be improved by using a model with more than one degree of freedom. Figure 10 displays the experimental function $\omega \mapsto \pi_{\mathrm{in}, \mathrm{norm}}^{\text {exp }}(\omega)$ (upper figure) and optimized model function $\omega \mapsto \pi_{\text {in,norm }}\left(\omega, \mathbf{w}^{\text {opt }}\right)$ (lower figure), for the seven values of $d / h$. A reasonable agreement can also be seen between the prediction and the experiments.

Furthermore, the results presented in these figures confirm a strong effect of the nonlinearity that allows the pumping energy phenomenon to be efficient 


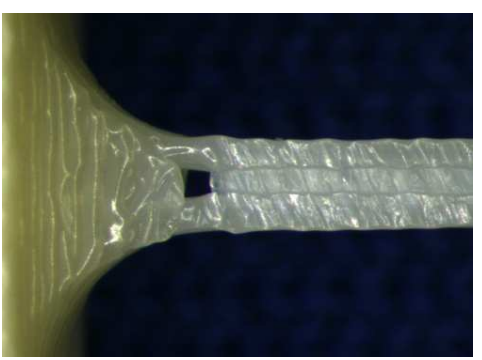

(a) Top view of the inclusion beam.

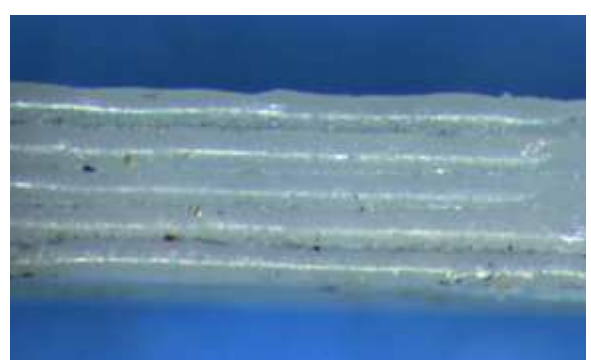

(b) Side view of the inclusion beam.

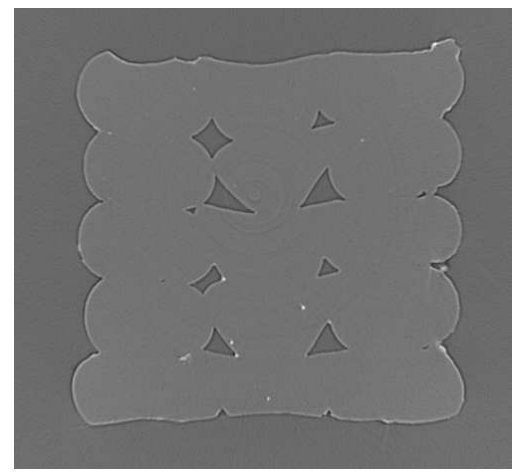

(c) View of a cross section of the inclusion beam.

Figure 8: Several views of the 3D-printing beam of the inclusion.

over a broader frequency band around the resonance frequency than for the linear case.

\section{Conclusions}

In this paper, we have presented the results related to the first stage of a work devoted to the design and the analysis of a nonlinear microstructured material to reduce noise and vibration at low frequencies. We have designed an inclusion that is a nonlinear absorber at macroscale, which has been manufactured with a $3 \mathrm{D}$ printing system. The dimension of this inclusion could easily be reduced with the same technology, in order to be inserted in a macro-structural element. A nonlinear dynamical model of the inclusion has been developed and its parameters have been identified with experiments. Both the predictions given by the nonlinear dynamical model and the experiments confirm that the pumping energy phenomenon is more efficient over a broad frequency band around the resonance frequency than for a linear 


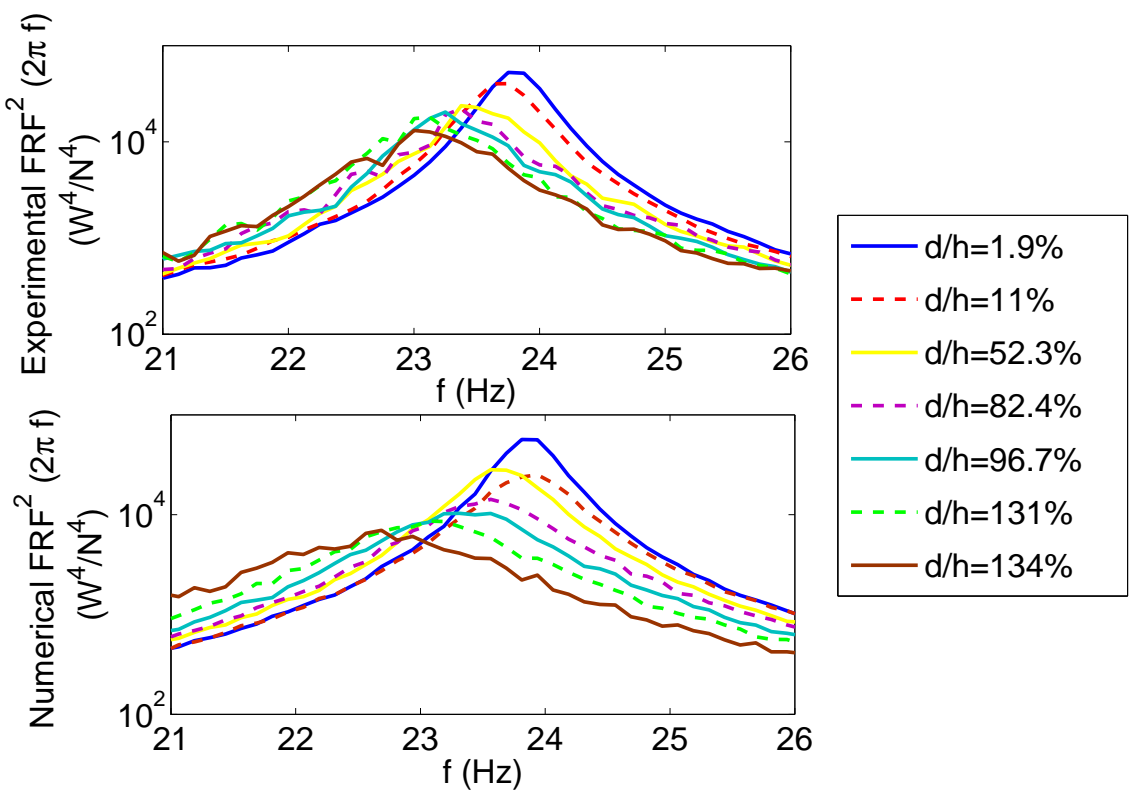

Figure 9: For the seven values of $d / h$, experimental function $\omega \mapsto \operatorname{FRF}^{\exp }(\omega)^{2}$ (upper figure) and optimized model function $\omega \mapsto \operatorname{FRF}\left(\omega, \mathbf{w}^{\mathrm{opt}}\right)^{2}$ (lower figure).

dynamical system. Note that the parametric experiments, which have been presented, allow for demonstrating the role played by the nonlinear geometrical effect absorption bandwidth, which is the expected phenomenon. The work in progress is the use of such inclusions for analyzing the attenuation of acoustics waves and vibrations in structural elements.

\section{Acknowledgements}

This work has benefited from a French government grant managed by ANR within the frame of the national program investments for the Future ANR-11-LABX-002-01.

\section{Bibliography}

[1] H. Frahm, Device for damping vibrations of bodies, United states patent office (1911) 1-9. 


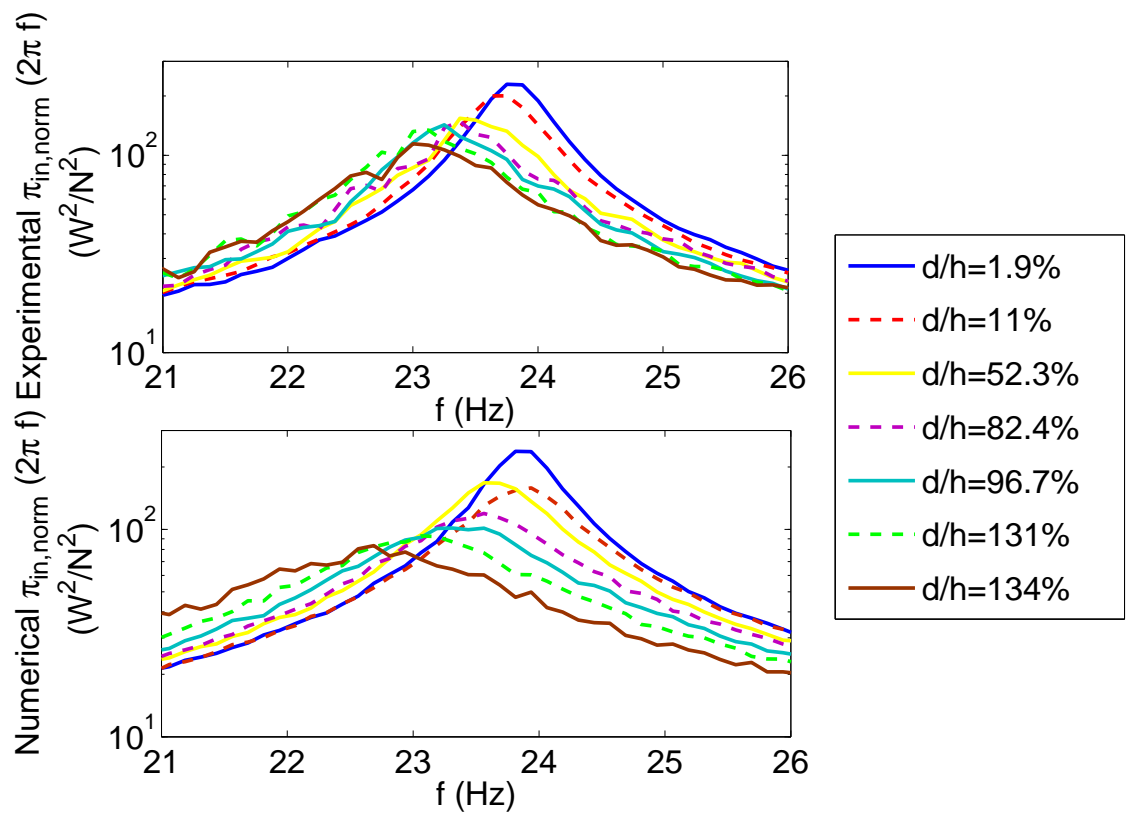

Figure 10: For the seven values of $d / h$, experimental function $\omega \mapsto \pi_{\text {in, norm }}^{\text {exp }}(\omega)$ (upper figure) and optimized model function $\omega \mapsto \pi_{\text {in,norm }}\left(\omega, \mathbf{w}^{\text {opt }}\right)$ (lower figure).

[2] M. Gutierrez Soto, H. Adeli, Tuned mass dampers, Archives of Computational Methods in Engineering 20 (4) (2013) 419-431.

[3] V. G. Veselago, The electrodynamics of substances with simultaneously negative values of $\epsilon$ and $\nu$, Soviet Physics 10 (1968) 509-514.

[4] Li, S. and Gao, X. L. (eds), Handbook of Micromechanics and Nanomechanics, Pan Stanford Publishing, 2013.

[5] D. Del Vescovo, I. Giorgio, Dynamic problems for metamaterials: Review of existing models and ideas for further research, International Journal of Engineering Science 80 (2014) 153-172.

[6] D. R. Smith, N. Kroll, Negative refractive index in left-handed materials, Physical Review Letters 85 (2000) 2933-2936.

[7] Y. Ding, Z. Liu, C. Qiu, J. Shi, Metamaterial with simultaneously negative bulk modulus and mass density, Physical Review Letters 99 (2007) 093904. 
[8] P. Sheng, J. Mei, Z. Liu, W. Wen, Dynamic mass density and acoustic metamaterials, Physica B 394 (2007) 256-261.

[9] Z. Yang, J. Mei, M. Yang, N. H. Chan, P. Sheng, Membrane-type acoustic metamaterial with negative dynamic mass, Physical Review Letters 101(20) (2008) 204301.

[10] S. H. Lee, C. M. Park, Y. M. Seo, Z. G. Wang, C. K. Kim, Acoustic metamaterial with negative density, Physics Letters A 373 (2009) 44644469.

[11] X. N. Liu, G. K. Hu, G. L. Huang, C. T. Sun, An elastic metamaterial with simultaneously negative mass density and bulk modulus, Applied Physics Letters 98 (2011) 251907.

[12] X. Wang, Dynamic behaviour of a metamaterial system with negative mass and modulus, International Journal of Solids and Structures 51 (2014) 1534-1541.

[13] H. Chen, C. T. Chan, Acoustic cloaking in three dimensions using acoustic metamaterials, Applied Physics Letters 91 (2007) 183518.

[14] X. Zhou, G. Hu, Analytic model of elastic metamaterials with local resonances, Physical Review B 79 (2009) 195109.

[15] M. I. Hussein, M. J. Frazize, Metadamping: an emergent phenomenon in dissipative metamaterials , Journal of Sound and Vibration 332 (2013) 4767-4774.

[16] R. Zhu, X. N. Liu, G. K. Hu, C. T. Sun, G. L. Huang, A chiral elastic metamaterial beam for broadband vibration suppression, Journal of Sound and Vibration 333 (2014) 2759-2773.

[17] Z. Yang, H. M. Dai, N. H. Chan, G. C. Ma, P. Sheng, Acoustic metamaterial panels for sound attenuation in the 50-1000 Hz regime, Applied Physics Letters 96 (2010) 041906.

[18] J.-L. Auriault, C. Boutin, Long wavelength inner-resonance cut-off frequencies in elastic composite materials, International Journal of Solids and Structures 49 (2012) 3269-3281.

[19] Y. Xiao, J. Wen, X. Wen, Sound transmission loss of metamaterialbased thin plates with multiple subwavelength arrays of attached resonators, Journal of Sound and Vibration 331 (2012) 5408-5423. 
[20] S. Varanasi, J. S. Bolton, T. H. Siegmund, R. J. Cipra, The low frequency performance of metamaterial barriers based on cellular structures, Applied Acoustics 74 (2013) 485-495.

[21] X. Wang, H. Zhao, X. Luo, Z. Huang, Membrane-constrained acoustic metamaterials for low frequency sound insulation, Applied Physics Letters 108(4) (2016) 041905.

[22] R. E. Roberson, Synthesis of a nonlinear dynamic vibration absorber, Portions of a dissertation submitted to the Department of Applied Mechanics, Washington University, in partial fulfillment of the requirements for the degree of Doctor of Philosophy. (1952) 205-220.

[23] R. Bouc, The power-spectral density of response for a strongly nonlinear random oscillator, Journal of Sound and Vibration 175 (1999) $317-331$.

[24] C. Soize, Stochastic linearization method with random parameters for SDOF nonlinear dynamical systems: Prediction and identification procedures, Probabilistic Engineering Mechanics 10 (3) (1995) Pages : 143152.

[25] S. Bellizzi, R. Bouc, Analysis of multi-degree of freedom strongly nonlinear systems with random input, Probabilistic Engineering Mechanics 14 (1999) 245-256.

[26] P. D. Spanos, I. A. Kougioumtzoglou, C. Soize, On the determination of the power spectrum of randomly excited oscillators via stochastic averaging: An alternative perspective, Probabilistic Engineering Mechanics 26 (2011) 10-15.

[27] C. Soize, Vibration damping in low-frequency range due to structural complexity. A model based on the theory of fuzzy structures and model parameters estimation, Computers and Structures 58 (1995) 901-915.

[28] O. Gendelman, L. I. Manevitch, A. F. Vakakis, R. MĆloskey, Energy pumping in nonlinear mechanical oscillators: part I-dynamics of the underlying hamiltonian systems, Journal of Applied Mechanics 68 (2001) $34-41$.

[29] A. F. Vakakis, O. Gendelman, Energy pumping in nonlinear mechanical oscillators: part II-resonance capture, Journal of Applied Mechanics 68 (2001) 42-48. 
[30] A. F. Vakakis, Shock isolation through the use of nonlinear energy sinks, Journal of Vibration and Control 9 (2003) 79-93.

[31] A. Carrella, M. J. Brennan, T. P. Waters, Static analysis of a passive vibration isolator with quasi-zero-stiffness characteristic, Journal of Sound and Vibration 301 (2007) 678-689.

[32] N. A. Alexander, F. Schilder, Exploring the performance of a nonlinear tuned mass damper, Journal of Sound and Vibration 319 (2009) 445462 .

[33] J. C. Ji, N. Zhang, Suppression of super-harmonic resonance response using a linear vibration absorber, Mechanics Research Communications 38 (2011) 411-416.

[34] J. C. Ji, Design of a nonlinear vibration absorber using three-to-one internal resonances, Mechanical Systems and Signal Processing 42 (2014) 236-246.

[35] L. D. Viet, N. B. Nghi, On a nonlinear single-mass two-frequency pendulum tuned mass damper to reduce horizontal vibration, Engineering Structures 81 (2014) 175-180.

[36] L. Cveticanin, M. KalamiYazdi, H. Askari, Z. Saadatnia, Vibration of a two-mass system with non-integer order nonlinear connection, Mechanics Research Communications 43 (2011) 22-28.

[37] D. Lavazec, G. Cumunel, D. Duhamel, C. Soize, Attenuation of mechanical vibrations at low frequencies by a nonlinear dynamical absorber, in: 23ème Congrès Français de Mécanique, Lille, France, 28 August -1 September 2017.

[38] B. D. Coleman, W. Noll, Foundations of Linear Viscoelasticity, Reviews of Modern Physics 33 (1961) 239-249.

[39] C. Desceliers, C. Soize, Non-linear viscoelastodynamic equations of three-dimensional rotating structures in finite displacement and finite element discretization, International Journal of Non-Linear Mechanics 39 (2004) 343-368.

[40] R. Y. Rubinstein, D. P. Kroese, Simulation and the Monte Carlo Method, Second Edition, John Wiley \& Sons, 2008. 
[41] A. Papoulis, Probability, Random Variables and Stochastic Processes, McGraw-Hill, New York, 1965.

[42] L. Guikhman, A. V. Skorokhod, The Theory of Stochastic Processes, Springer Verlag, 1979.

[43] M. B. Priestley, Spectral Analysis and Time Series, Academic Press, New York, 1981.

[44] M. Shinozuka, Simulation of multivariate and multidimensional random processes, Journal of the Acoustical Society America 49 (1971) 357-367.

[45] F. Poirion, C. Soize, Numerical methods and mathematical aspects for simulation of homogeneous and non homogeneous Gaussian vector fields, in: P. Krée, W. Wedig (Eds.), Probabilistic Methods in Applied Physics, Springer-Verlag, Berlin, 1995, pp. 17-53.

[46] L. Verlet, Computer "experiments" on classical fluids. I. Thermodynamical properties of Lennard-Jones molecules, Physical Review 159 (1) (1967) 98-103.

[47] E. Hairer, C. Lubich, G. Wanner, Geometric numerical integration illustrated by the Störmer/Verlet method, Acta Numerica 12 (2003) 399450 .

[48] C. Soize, I. E. Poloskov, Time-domain formulation in computational dynamics for linear viscoelastic media with model uncertainties and stochastic excitation, Computers and Mathematics with Applications 64 (11) (2012) 3594-3612. 